\title{
Is Genetic Involve in The Outcomes of Contact Reactions Between Parent And Offspring And Between Siblings of The Coral Pocillopora damicornis?
}

\author{
Diah Permata Wijayanti ${ }^{1}$ and Michio Hidaka ${ }^{2}$ \\ ${ }^{1}$ Marine Science Department, Universitas Diponegoro, \\ Jl. Prof. Soedarto SH., Tembalang, Semarang, Indonesia 50275 \\ ${ }^{2}$ Chemistry, Biology and Marine Science Department, \\ University of the Ryukyus, Nishihara, Okinawa 903-0213, Japan \\ Email: diahpermata@mail.com
}

\begin{abstract}
Various allogeneic responses have been characterized in stony corals. The responses were thought to be genetically control. However, very little is known about the genetic mechanism of allorecognition in corals. Therefore, the contact reaction between parent-offspring and between siblings of the coral Pocillopora damicornis has been studied. Three types of contact reactions were observed: between young colonies, between adult and young colonies, and between adult colonies. Siblings, which were primary polyps or young colonies derived from the same broodparent, invariably fused. All but one pair of parent-offspring grafts exhibited fusion. However, some pairs of young colonies derived from the same two broodparents showed a different contact response than did the broodparents when their branch tips were paired. When the same pairs of the young colonies were repeated to contact at different age, most pairs yielded the same results. Together with the findings that young colonies were able to recognize their partner less than 1 month after the initial contact, we suggest that contact reaction in coral and time needed for the stable reaction are most probably under genetic control.
\end{abstract}

Keywords: coral, young colonies, reproduction, parent-offspring relationship, histocompatibility

\section{Introduction}

Marine sessile colonial invertebrates have ability to distinguish self from non-self. Allogeneic reactions are well described in corals that result in either fusion between contacting allogeneic partners in a wide array of histoincompatible outcomes or terminated in various 'rejection' responses (Chadwick-Furman and Rinkevich, 1994; Frank et al., 1997; Hidaka et al., 1997; Amar et al., 2008), as in other sessile invertebrates such colonial ascidians, bryozoa, hydroids, and sponges. However, the genetic mechanism that controls the response has been described only in the ascidian Botryllus (Oka and Watanabe, 1957; 1960; De Tomaso et al., 2005) and in the hydroid Hydractinia (Cadavid et al., 2004).

In adult corals, various allogeneic responses have been described (reviewed in Chornesky, 1991). The responses were thought to be genetically control (Hunter, 1985; Amar and Rinkevich, 2010; Hennige et al., 2014; Schweinsberg et al., 2015; Rinkevich et al., 2016). While in juvenile, maturity state during initial contact was proposed as another primary determinant of the contact reaction (Hidaka, 1985; Frank et al., 1997; Barki et al., 2002; Nozawa and Loya, 2005; Amar et al., 2008). Much-slower maturation of the allorecognition system was suggested for Echinophyllia aspera juveniles (Nozawa and Hirose, 2011) as well as slower responses showed by larvae of spawner type corals Acropora millepora of the Great Barrier Reefs (PuillStephan et al., 2012). However, the genetic mechanism controlling the responses has not been investigated.

Pocillopora damicornis is one of the most widespread corals. It is highly variable, not only in morphology (Veron and Pichon, 1976) but also in reproductive mode (Harrison and Wallace, 1990). $P$. damicornis is known to reproduce both of asexual and sexual modes (Harrison and Wallace, 1990). The coral reproduced through parthenogenetic planulae (Stoddart, 1983), also as hermaphroditic brooder over most of its range in the Indo-Pacific Ocean (Diah Permata et al., 2000) and hermaprodictic broadcasting (Ward, 1992; Miller and Ayre, 2004; Schmidt-Roach et al., 2012).

It was generally agreed that the best method to determine the genetic background of histocompatibility is through inheritance studies (Grosberg, 1988). The purpose of this study was to investigate contact reactions between siblings, between parent and its offspring in an attempt to understand genetic mechanism that controls the contact reaction in coral. 
In colonial corals, clonemates always fuse with each other in histocompatibility assays (Hildemann et al. 1977; 1980). Thus, pairs of young colonies derived from asexually generated planulae should show the same contact response, which should also be the same as that exhibited between their source colonies. If two colonies do not fuse, they are genetically different. Although the control mechanism for the histoincompatibility response of coral is not known, a histoincompatible response between primary polyps or young colonies and their sibling colonies or broodparent indicates genetic differences between them. We also investigated whether pairs of young colonies derived from the same two source colonies always showed the same contact response to each other and whether they displayed the same response as that shown by their source colonies to each other. The contact reaction was observed for over 6 months in order to avoid missing incompatible responses due to possible lack of histocompatibility system in young colonies. If various outcomes were observed from three types of contact reactions most probably the type of contact reactions produced is genetically control.

\section{Materials and Methods}

\section{Collection of corals and planulae}

Colonies of Pocillopora damicornis (approximately $10 \mathrm{~cm}$ in diameter) were collected from two sites, Bise (PB and PBK) and Motobu (MT), in northern Okinawa Island, and two sites, Zamami Island (ZR) and Tokashiki Island (TS), in the Kerama Islands region (Figure 1.). Colonies were transported to Sesoko Station, Tropical Biosphere Research Center, University of the Ryukyus, Okinawa, Japan, and kept individually in 2-I plastic buckets supplied with unfiltered running seawater.

Planulae were collected around the first quarter-moon by filtering overflowing seawater through planula collectors made of 60 - or $180-\mu \mathrm{m}$ nylon mesh. The collected planulae were placed in another container sealed on the bottom with 180 $\mu \mathrm{m}$ nylon mesh. The inner side wall and bottom of the container were covered with plastic sheets (Kokuyo, tracing film \#200) so that planula settled on them (Hidaka, 1985). Three types of contact experiments were performed: between young colonies, between adult and young colonies, and between adult colonies (Permata and Hidaka, 2005).

\section{Contact experiments between young colonies}

Contact responses between sibling young colonies (colonies derived from planulae from the same colony) and those between non-sibling young colonies (colonies derived from planulae from different colonies) were studied. Primary polyps (settled and metamorphosed planulae) were put in contact with each other 3 days to 2 weeks after planulation. Two plastic sheets, each with a primary polyp, were juxtaposed on a glass slide so that the partners were as close as possible to each other. Since primary polyps began to bud within a few days after settlement, most individuals had grown into young colonies when the contact reaction was recorded. Some pairs of young colonies were coupled 3-4 months after planulation to test whether the age of young colonies affected the contact reaction.

Two series of experiments were conducted with primary polyps (young colonies). In the first series, 48 pairs of siblings were prepared using primary polyps derived from three source colonies. The polyp pairs were put in contact 3 days to 2 weeks after planulation. The contact responses of 43 pairs were recorded; five pairs were discarded due to the death of one or both of the colonies.

The second experimental series, contact reactions were recorded for 139 pairs (44 siblings and 95 non-siblings) of more than 200 prepared pairs of siblings and non-siblings; the rest were omitted because of the death or detachment of one or both partners before they established contact or because a pair failed to establish significant contact. Detailed of pairs prepared for contact reaction between young colonies can be observed at Table 1, respectively.

\section{Contact experiments between adult and young colonies}

Contact experiments between young colonies and their source colonies (broodparent) as well as those between young colonies and unrelated adult colonies (non-parent) were studied. Small branch tips approximately 3-5 $\mathrm{mm}$ long were cut from adult colonies and fixed with silicone tubing and a nylon thread clamp to plastic sheeting on a glass slide. The branch tips were allowed to recover for 1 week before the contact experiments began. Young colonies ( 3 days to 2 weeks post-planulation) were then positioned several millimeters from the branch tips. The outcome was recorded after the growing edges of both tissues contacted each other. The branch tips were cut about the same size as the young colonies at the time of contact. Number of samples observed was presented at Table 2.

\section{Contact experiment between adult colonies}

To investigate whether young colony pairs showed the same contact response as that displayed by pairs of their adult source colonies, we 
Table 1. Contact reactions between young colonies of Pocillopora damicornis. The number of pairs showed each type of contact response is shown for each combination. F, fusion; NF, non-fusion; ICF, incompatible fusion. For the secondary outcomes, the number of the pairs that transformed from fusion to incompatible fusion ( $F>I C F)$; fusion to non-fusion $(\mathrm{F}>\mathrm{NF})$; incompatible fusion to disconnection (ICF>DC); or non-fusion to disconnection (NF>DC) is shown.

\begin{tabular}{|c|c|c|c|c|c|c|c|c|}
\hline \multirow{2}{*}{ Exp. ${ }^{a}$} & \multirow{2}{*}{ Combination ${ }^{b}$} & \multirow[b]{2}{*}{$\mathrm{F}$} & \multicolumn{3}{|c|}{ Initial outcomes } & \multicolumn{2}{|c|}{ Second outcomes } & \multirow[b]{2}{*}{$\mathrm{F}>\mathrm{DC}$} \\
\hline & & & NF & ICF & $\mathrm{F}>\mathrm{ICF}$ & $\mathrm{F}>\mathrm{NF}$ & ICF $>$ DC & \\
\hline & Siblings & & & & & & & \\
\hline \multirow[t]{4}{*}{ I } & PB01-PB01 & 28 & & & & & & \\
\hline & PB02-PB02 & 5 & & & & & & \\
\hline & PB03-РB03 & 10 & & & & & & \\
\hline & Total & 43 & & & & & & \\
\hline \multirow[t]{12}{*}{$\|$} & PB07-PB07 & $2+2 *$ & & & & & & \\
\hline & PB08-PB08 & $3+2 *$ & & & & & & \\
\hline & PB09-PB09 & $3+2 *$ & & & & & & \\
\hline & PB10-PB10 & $3+4 *$ & & & & & & \\
\hline & PB11-PB11 & 2 & & & & & & \\
\hline & PB12-PB12 & 5 & & & & & & \\
\hline & PBK1-PBK1 & $5+2 *$ & & & & & & \\
\hline & PBK2-PBK2 & $3+1 *$ & & & & & & \\
\hline & ZR3-ZR3 & 2 & & & & & & \\
\hline & MT5-MT5 & 3 & & & & & & \\
\hline & Total & $31+13 *$ & & & & & & \\
\hline & Non siblings & & & & & & & \\
\hline \multirow[t]{17}{*}{ II } & PB08-PB09 & $2+2 *$ & & & & & & \\
\hline & PB08-PB10 & $4+2 *$ & & & & & & \\
\hline & PB09-PB10 & $6+5 *$ & & & & & & \\
\hline & PB08-PBK2 & 6 & & & & & & \\
\hline & PB10-PBK2 & 6 & & & & & & \\
\hline & PB07-PB08 & & $6+2 *$ & & & & & 6 \\
\hline & PB07-PB10 & 1 & $5+1 *$ & & & & & 1 \\
\hline & PB08-PBK1 & & $5+1 *$ & 1 & & & 1 & 2 \\
\hline & PB09-PBK1 & & 6 & & & & & 5 \\
\hline & PB10-PBK1 & & $1+2 *$ & 1 & & & 1 & $1 *$ \\
\hline & PBK1-PBK2 & & 3 & & & & & 2 \\
\hline & PBK1-ZR3 & & 6 & & & & & 3 \\
\hline & PB07-ZR3 & & $5+1 *$ & & & & & 4 \\
\hline & PB08-ZR3 & 6 & $1 *$ & & 4 & & & \\
\hline & PB09-ZR3 & 2 & & 2 & & 2 & 2 & \\
\hline & PB10-ZR3 & 3 & & $1 *$ & 2 & & & \\
\hline & Total & $36+9 *$ & $37+8^{*}$ & $4+1^{*}$ & 6 & 2 & 4 & $23+1 *$ \\
\hline
\end{tabular}

axp.I and II were performed for six months.

${ }^{b}$ Combinations of young colonies are expressed as combinations of source colonies from which planulae were derived. The letters, PB, PBK, ZR, and MT indicate sites where the source colonies were collected; PB and PBK, Bise; ZR, Zamami; MT, Motobu. *Represents pairs of young colonies that were grafted at 3 to 4 months after planulation.

assayed small branches of the adult colonies. Branch tips were prepared as described above and positioned several millimeters from each other to allow for growth. The outcome was recorded after the growing edges of both tissues contacted other.

Contact reactions were recorded for 52 pairs (eight isografts and 51 allografts of 31 combinations) out of a total of 70 prepared pairs (12 isografts and 58 allografts of 32 combinations), with the rest omitted because of the death or loss of a partner before contact. In eight of the 32 allogeneic combinations, contact responses were also evaluated for young-young and adult-young pairs so that we could compare the outcomes of the three types of contact reactions using the same combinations. See Table 3.

\section{Record of contact reactions}

The glass slides on which the grafted pairs were mounted were labeled and held vertically in slide holders. The pairs were maintained in an indoor tank supplied with unfiltered running seawater and exposed to natural light through a glass roof and windows. All pairs were observed and 
photographed under a stereomicroscope at 1-2week intervals for the first 2 months and once a month thereafter.

The contact responses were classified as fusion after Chadwick-Furman and Rinkevich (1994), Hidaka et al. (1997), Frank et al. (1997), Nozawa and Loya (2005) and Amar et al. (2008), and incompatible fusion (Hidaka et al., 1997; Nozawa and Loya, 2005) (see Box 1 for details). A disconnection response occurred when pairs became separated as tissue at the interface died. We observed contact responses between young colonies for a 6-month period in order to reveal incompatible responses veiled by the possible lack of a histoincompatibility system in young colonies (Hidaka 1985; Hidaka et al., 1997; Frank et al., 1997; Nozawa and Loya, 2005).

\section{Results and Discussion}

\section{Contact reactions between young colonies}

Typical outcomes of contact experiments between young colonies are shown in Figure 2. The numbers of pairs that showed each reaction are summarized in Table 1. Siblings, i.e., young colonies derived from the same broodparent, always fused with each other (Table 1). Zooxanthellae were distributed evenly across the interface area of fusion pairs (Figure 2A). In the first series of experiments, 43 pairs of siblings derived from three source colonies displayed a fusion response. In the second series of experiments, siblings were brought into contact at different times after planulation. All siblings fused, regardless of the age (2 weeks or 3-4 months after planulation) at which they were assayed.

Pairs of non-sibling young colonies, which came from different source colonies, showed either fusion, non-fusion, or incompatible fusion (Figure 2B, $C$, D.). Fusion in non-sibling young colonies was similar to fusion among siblings (Figure 2B.). In nonfused pairs, a suture or a white line demarcated the tissues of the paired colonies (Figure 2C.). Both colonies secreted skeleton at the interface to form a skeletal ridge. The skeletal ridge usually grew vertically at first but later inclined toward one of the pair, resulting in overgrowth of one partner by the other. However, complete overgrowth or killing of the opponent was not observed during the 6-month observation period. In incompatibly fused pairs, tissues of the paired colonies were continuous, but the interface region was constricted, and a white zone with few zooxanthellae was observed at the contact region (Figure 2D.), as described previously

Table 2. Contact reactions between adult and young colonies of Pocillopora damicornis. The number of pairs showed each type of contact response is shown for each combination. F, fusion; NF, non-fusion; ICF, incompatible fusion. For the secondary outcomes, the number of the pairs that transformed from fusion to incompatible fusion (F>ICF) or nonfusion to disconnection $(\mathrm{NF}>\mathrm{DC})$ is shown.

\begin{tabular}{|c|c|c|c|c|c|c|c|}
\hline \multirow{2}{*}{ Exp. $^{a}$} & \multirow{2}{*}{ Combinationb } & \multicolumn{3}{|c|}{ Initial outcomes } & \multicolumn{3}{|c|}{ Secondary outcomes } \\
\hline & & $\mathrm{F}$ & NF & ICF & $\mathrm{F}>\mathrm{ICF}$ & $\mathrm{ICF}>\mathrm{DC}$ & $\mathrm{NF}>\mathrm{DC}$ \\
\hline & Parent-offspring & & & & & & \\
\hline \multirow[t]{3}{*}{ I } & PB02-PB02 & 1 & & & & & \\
\hline & PB03-PB03 & 1 & & & & & \\
\hline & Total & 2 & 0 & 0 & 0 & 0 & 0 \\
\hline \multirow[t]{8}{*}{ II } & MT5-MT5 & 2 & & & & & \\
\hline & PB07-PB07 & $2+2 *$ & & 1 & & 1 & \\
\hline & PB08-PB08 & $3+1 *$ & & & & & \\
\hline & PB09-PB09 & $4+1 *$ & & & & & \\
\hline & PB10-PB10 & $4+1 *$ & & & & & \\
\hline & PB12-PB12 & 1 & & & & & \\
\hline & Total & $16+5 *$ & 1 & 0 & 0 & 1 & 0 \\
\hline & Unrelated adult-young & & & & & & \\
\hline \multirow[t]{7}{*}{ ॥ } & PB08-PB07 & 2 & & & & & \\
\hline & PB09-PB08 & 2 & & & & & \\
\hline & PB07-ZR3 & & 2 & & & & 1 \\
\hline & PB09-ZR3 & 1 & & & & & \\
\hline & PB10-ZR3 & 2 & & & 1 & & \\
\hline & MT5-PB10 & & 2 & & & & \\
\hline & Total & 7 & 4 & 0 & 1 & 0 & 1 \\
\hline
\end{tabular}

${ }^{\mathrm{a}}$ Exp.I and II were performed for six months.

bCombination between young colonies and parents are expressed as combinations of source colonies from which the pairs were derived. The letters, PB, ZR, and MT indicate sites where the source colonies were collected; PB, Bise; ZR, Zamami; MT, Motobu. *Represents pairs of parent-offspring that were grafted after offspring reached 3 months old after planulation. 


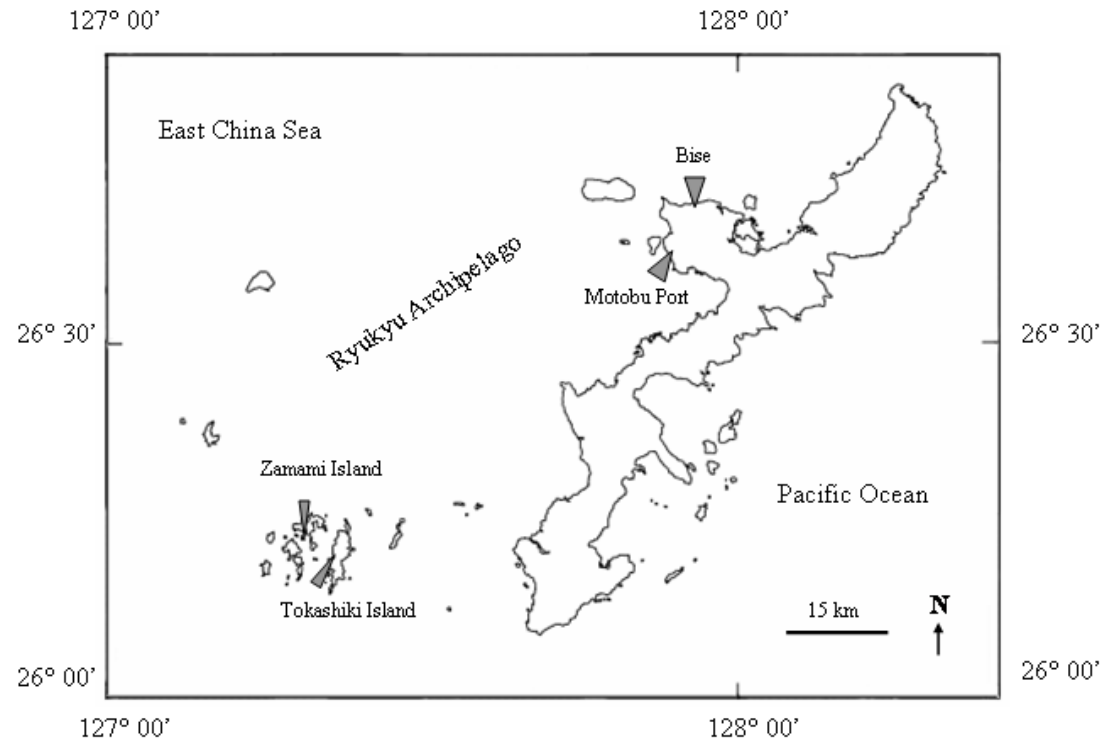

Figure 1. Ryukyu Archipelago, showing the location of the four Pocillopora damicornis sampling sites.

Table 3. Contact reactions between adult colonies of Pocillopora damicornis. The number of pairs showed each type of contact response is shown for each combination. F, fusion; NF, non-fusion; ICF, incompatible fusion. Secondary outcomes were observed only in allogeneic pairs. For the secondary outcomes, the number of the pairs which transformed from fusion to non-fusion ( $\mathrm{F}>\mathrm{NF}$ ) is shown.

\begin{tabular}{|c|c|c|c|c|c|}
\hline & \multirow{2}{*}{ Combinationa } & \multicolumn{3}{|c|}{ Initial outcomes } & \multirow{2}{*}{$\begin{array}{c}\text { Second outcomes } \\
F>N F\end{array}$} \\
\hline & & $\mathrm{F}$ & NF & ICF & \\
\hline \multirow[t]{7}{*}{ PB } & PB08-PB07 b & & 2 & & \\
\hline & PB07-PB10 b & & 2 & & \\
\hline & PB08-PB10 b & 2 & & & \\
\hline & PB09-PB10 b & 3 & & & \\
\hline & PB13-PB14 & 2 & & & \\
\hline & PB13-PB15 & 2 & & & \\
\hline & PB14-PB15 & 2 & & & \\
\hline \multirow[t]{7}{*}{ MT } & MT1-MT2 & 2 & & & \\
\hline & MT1-MT3 & 1 & & & \\
\hline & MT1-MT6 & 1 & & & \\
\hline & MT2-MT3 & 2 & & & \\
\hline & MT2-MT6 & 2 & & & \\
\hline & МT3-МT5 & 2 & & & \\
\hline & МT3-MT6 & 2 & & & \\
\hline \multirow{4}{*}{ PB-MT } & PB06-MT5 & & 2 & & \\
\hline & РB09-MT5 & & 1 & & \\
\hline & PB13-MT2 & & 2 & & \\
\hline & PB14-MT2 & & 2 & & \\
\hline \multirow[t]{4}{*}{ PB-ZR } & PB06-ZR4 & 1 & 1 & & \\
\hline & PB07-ZR3 b & & 1 & & \\
\hline & PB08-ZR3 b & 2 & & & \\
\hline & PB09-ZR3 b & 1 & & & \\
\hline PB-TS & PB06-TS3 & 2 & & & \\
\hline \multirow[t]{2}{*}{ MT-TS } & MT2-TS3 & & & 2 & \\
\hline & MT3-TS3 & 2 & & & 1 \\
\hline \multirow[t]{2}{*}{ MT-ZR } & MT2-ZR5 & & 1 & & \\
\hline & MT3-ZR4 & 1 & & & \\
\hline \multirow[t]{4}{*}{ TS-ZR } & TS3-ZR4 & & 1 & & \\
\hline & TS3-ZR5 & & 1 & & \\
\hline & TS4-ZR2 & & 1 & & \\
\hline & Total & 32 & 17 & 2 & 1 \\
\hline
\end{tabular}

a The letters, PB, ZR, MT, and TS indicate sites where the source colonies were collected; ZR, Zamami; PB, Bise; MT, Motobu; TS, Tokashiki. brepresents that non-siblings assays were performed using young colonies derived from the same source colonies. 

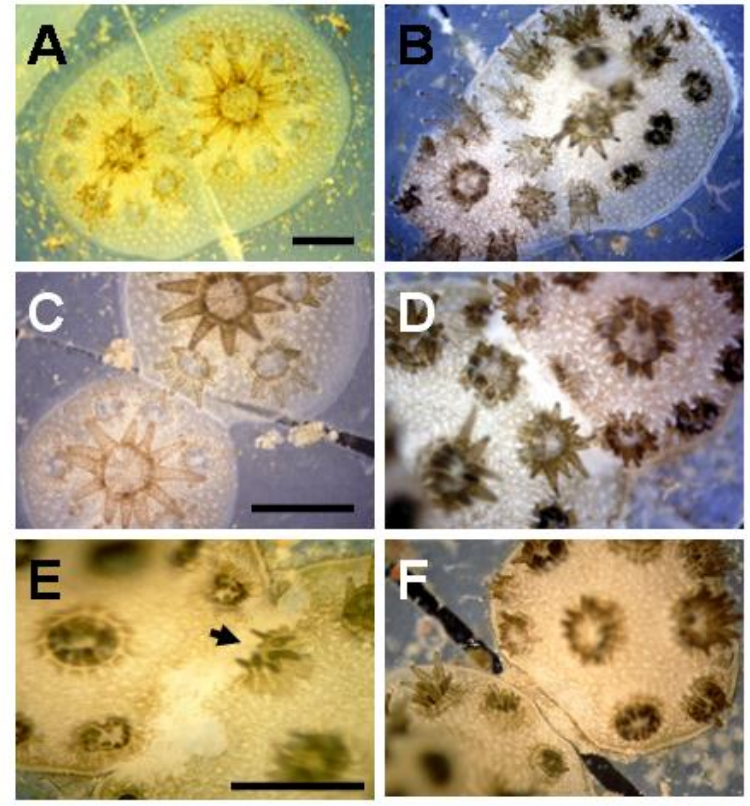

Figure 2. Contact reactions between young Pocillopora damicornis colonies. A Fused pair of siblings derived from the same broodparent (PBO1) 24 days after the contact experiment began. B Fusion of non-siblings from colonies PB10 and ZR3 (52 days). C Non-fused pair of non-siblings from colonies PB07 and ZR3 (24 days). D Incompatible fusion of non-siblings from colonies PBK1 and PB08 (52 days). E Polyp resorption in an incompatibly fused pair from colonies PB08 and PB07 (52 days). A polyp (arrowhead) being absorbed. At the end of experiment the pair showed Disconnection. F Disconnection of a nonsibling pair derived from PBK1 and PB08 (56 days). Scale bar $=10 \mathrm{~mm}$ (Scale in $C$ applies to $B$, D, F).

(Hidaka et al., 1997; Nozawa and Loya, 2005). In some pairs, the white zone was broad, and the polyps located close to the zone were partially absorbed by the white zone (Figure 2E). Resorp polyp was observed in 6 out of 24 pairs that showed Disconnection, which occurred when the pairs showing non-fusion or incompatible fusion became separated after tissue at the boundary died (Table 1). In many pairs, the dead area extended several millimeters and was eventually occupied by filamentous algae (Figure 2F.).

Replicate pairs of non-sibling young colonies derived from the same two source colonies usually displayed identical contact responses (Table 1.). Of the 11 combination pairs of non-siblings derived from source colonies at Bise, pairs of the first five combinations fused regardless of their age at the time of assay (2 weeks or 3-4 months after planulation), while pairs of the other six combinations showed a non-fusion response regardless of the age of the young colonies (Table 1.). For remaining five combinations, one partner was derived from Bise and the other from Zamami source colonies. Pairs of the first two combinations did not fuse, while those of the remaining three did. Pairs involving colony PBO7 or PBK1 as a partner did not fuse, whereas pairs from other colonies did (Table 1). However, in some cases, pairs of the same combination showed different contact responses (Figure 3, Table 1.). In the PB07-PB10 colony combination, one pair showed stable fusion while the other five pairs exhibited non-fusion. In the PB08-PBK1 and PB10-PBK1 combinations, both non-fusion and incompatible fusion was observed. In PB09-ZR3, two pairs showed fusion while the other two pairs showed incompatible fusion. All four pairs later transformed into non-fusion (Figure 3.). In PB08-ZR3 and PB10-ZR3, two-thirds of the pairs transformed to incompatible fusion within the 6month observation period (Figure 3 , Table 1.). In these two combinations (PB08-ZR3 and PB10-ZR3), pairs that were brought into contact 3-4 months after planulation showed incompatible fusion or nonfusion, while pairs assayed at an earlier age initially showed fusion. Thus, the fusion observed in the three combinations with ZR3 as one partner appeared to be temporary. With the exception of the PB08-ZR3 and PB10-ZR3 combinations, non-sibling pairs that were assayed when both partners were 3 4 months old showed a contact reaction identical to that of younger colonies in the same partnering.

\section{Contact reactions between adult and young colonies}

Except for one PB07-PB07 combination, all parent-offspring pairs fused (Table 2). When tissues from each colony came into contact, a narrow tissue bridge initially connected the colonies. It soon became wider, and complete fusion occurred (Figure $4 \mathrm{~A})$. We observed fusion in 18 pairs that were brought into contact when the young colonies were 3 days to 2 weeks old, and in five pairs that were 3-4 months old upon contact. PB07-PB07, the one pair that did not fuse, showed incompatible fusion (Table 2). In this pair, the tissues appeared to be continuous, but a white zone occurred at the interface (Figure 4B). Four months later, the colonies disconnected. We re-grafted the same young colony onto another small branch isolated from the source colony. The re-grafted pair showed non-fusion after 62 days.

Pairs of adult colonies and unrelated young colonies exhibited either fusion or non-fusion (Table 2.). Seven pairs of four combinations fused, although one pair (PB10-ZR3) switched to incompatible fusion after 56 days. Two combinations (PB07-ZR3 and MT5-PB10) did not fuse (Figure 4C.). 
PB08 adult colonies and young PB07 colonies unexpectedly fused, while young PB07-PB08 colony pairs never fused.

\section{Contact reactions between adult colonies}

All isogeneic pairings of adult colonies resulted in fusion (eight pairs using eight colonies). Allogeneic pairs showed either fusion, non-fusion, or incompatible fusion (Table 3.). Fusion began 20-50 days after the start of the contact experiment and persisted until the end of the 5-month observation period (Figure 5A.). When colonies collected from the same site (Bise or Motobu) were paired, all but four pairs involving colony PB07 fused. Pairings of colonies from different sites resulted in non-fusion for four combinations (seven pairs) of Bise and Motobu colonies as well as three combinations (three pairs) of Tokashiki and Zamami colonies. In other combinations (Bise-Zamami, Motobu-Tokashiki, Motobu-Zamami), some pairs fused, while other pairs exhibited non-fusion, and two pairs of the MT2TS3 combination displayed incompatible fusion (Figure 5B.). When allogeneic pairs fused, the fusion was stable and persisted until the end of the experiment, except for one MT3-TS3 pair, which changed to non-fusion 56 days after the contact experiment began (Table 3.). One fusion and one

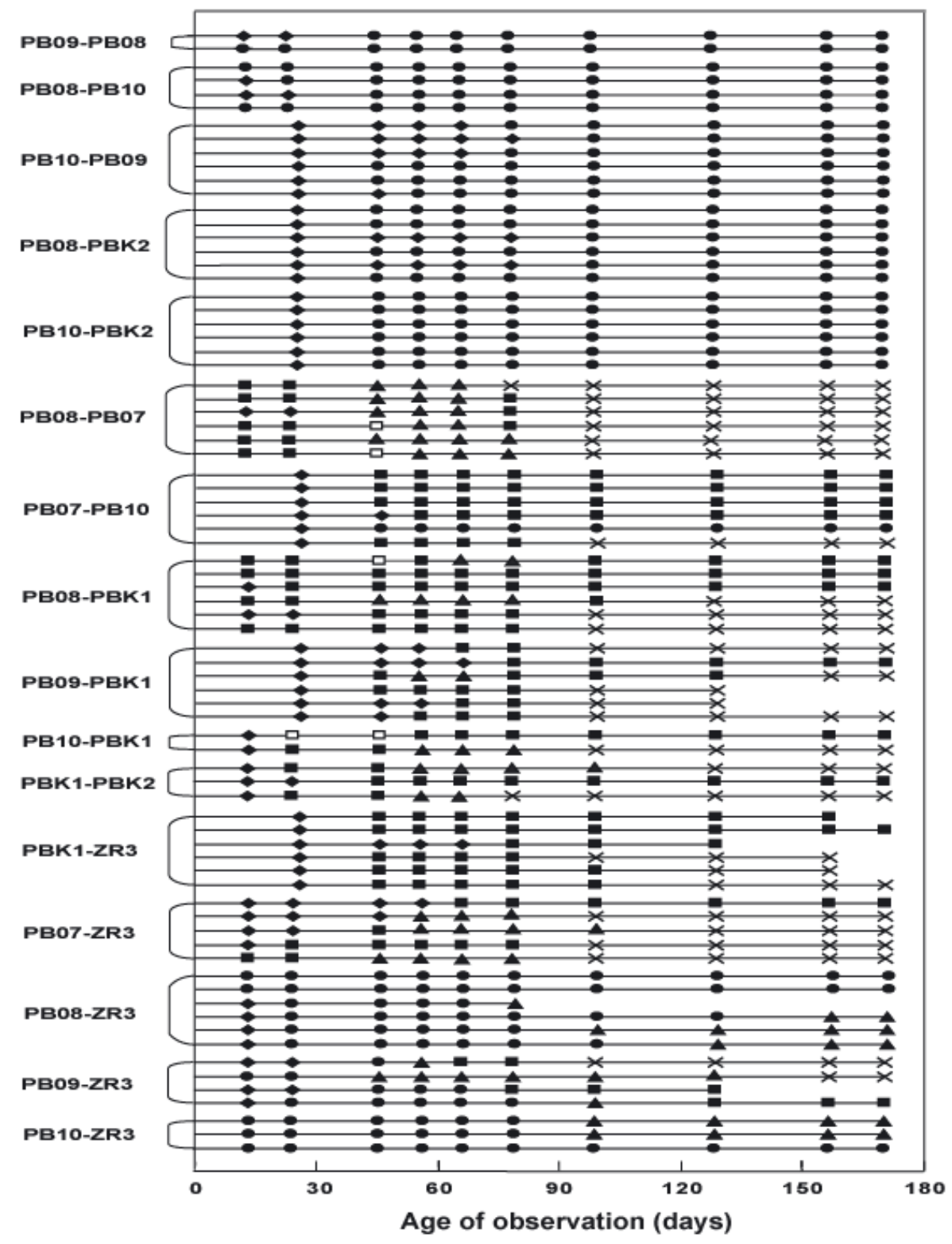

Figure 3. Dynamics of contact reactions between non-sibling pairs of young Pocillopora damicornis colonies. Each horizontal line represents a grafted pair, and symbols on the line represent the type of contact response. $\bullet$, fusion; $\boldsymbol{\Lambda}$, incompatible fusion; $\boldsymbol{\bullet}$, non-fusion; $\square$, incompatible fusion/non-fusion; $\times$, disconnection; tissue contact but without a clear response. Each pair was observed repeatedly: the first and last symbol on each line indicate the age of the pair at initial contact and at the last observation 
non-fusion were observed for the PB06-ZR4 combination. A suture formed at the interface of non-fused pairs (Figure 5.). None of the non-fused pairs disconnected; in most cases, one overgrew the other.

\section{Comparison of the course of contact reaction among young-young, young-adult, and adult-adult pairs}

To investigate whether pairs involving young colonies showed delayed response than pairs of adults, the course of the contact reaction was observed. Fusion reaction occurred $100 \%$ in siblings as in isogeneic pairs (Table 1.) while parent-offspring pairs showed $95.8 \%$ fusion since 1 pair displayed a disconnection (Table 2.). Complete fusion (all grafts recorded display a fusion reaction) in sibling pairs was observed at 14-129 days upon contact while in isogeneic pairs, complete fusion occurred within 2041 days. Parent-offspring pairs showed a complete fusion after 23-99 days. However, time scale of fusion among pairs of three different assays of contact reaction did not vary significantly (ANOVA, $F$ $=0.23, \mathrm{df}=1,36, P>0.1$ ).

Among non-siblings pairs, $38.9 \%$ pairs showed fusion reaction while $24.2 \%$ pairs showed non-fusion (Table 1.). However, contrast to the previous study (Hidaka et al., 1997) only $7.4 \%$ pairs showed incompatible fusion while disconnection was recorded in $29.5 \%$ pairs. Differ to the previous

studies on Hawaiian populations (Hidaka, 1985), the present study observed a high percentage of allogeneic fusion. Fusion was observed in $60.8 \%$ pairs, while $35.3 \%$ pairs displayed non-fusion, and only $3.9 \%$ pairs showed incompatible fusion reaction.

Though, pairs of non-sibling displayed more variable reactions compare to allogeneic pairs before a stable outcome was observed, fusion apparently was the most stable reaction in nonsiblings pairs (Table 1 ) while in adult allograft pairs, non-fusion appear as another stable reaction in addition to fusion reaction(Table 3 ). In non-siblings pair complete fusion was observed at 14-75 days after the commencement of contact reaction while in allograft pairs took 12-75 days upon contact. Nonfusion reaction can be observed after 20-56 days (Figure 3).

Comparison of contact experiments with youngyoung, young-adult, and adult-adult pairs

To investigate whether pairs of young colonies showed the same contact response as that demonstrated by pairs of their adult source colonies, we performed contact experiments with young-young, adult-young, and adult-adult pairs in the same combinations, using four colonies (PB07, 08, 09, 10) from Bise and one colony (ZR3) from Zamami (Table 4 ). We observed contact responses between young colonies and their adult source colonies in seven allogeneic and four isogeneic combinations. In general, pairs of young colonies showed the same response as did pairs of adult colony branch tips (Table 4). However, there were exceptions. In PB07PB07 combinations, one parent-offspring pair showed incompatible fusion, while the other four parent-offspring pairs as well as young-young and adult-adult pairs exhibited fusion. In PB07-PB10 combinations, only one young colony pair fused, while the other young-young pairs and adult-adult pairs did not. In PB07-PB08 combinations, parentoffspring pairs fused, while young-young and adultadult pairs did not. When colonies collected from Bise (PB08, 09) were combined with a colony from Zamami (ZR3), young-young pairs fused only temporarily, while the fusion of adult pairs was stable for the 5-month observation period.

The study showed that sibling pairs always fuse with each other (Table 1.). All 74 pairs of siblings from 13 source colonies fused, as did 13 pairs that were brought into contact at a later stage (3-4 months after planulation). Moreover, with the one exception, all 16 parent-offspring pairs, together with an additional five pairs that were brought into contact 3-4 months after planulation, fused. The exception (PB07-PB07) had an incompatible fusion response, but four other pairs derived from PB07 fused.

Several explanations are possible as to why larvae from the same broodparent invariably fused, as did almost all offspring-parent pairs. First, planulae may be produced asexually and thus be genetically identical to each other and to their broodparent, as suggested by Stoddart (1983), Ayre and Miller (2004) and recently Yeoh and Dai (2009). The latest authors reported only 35 larvae have genotypically distinct from 200 larvae randomly genotyped. The high proportion of clonal was found in both selected population. Second, if the fusibility of $P$. damicornis is controlled by a genetic mechanism similar to the colony-specificity locus in colonial ascidians (De Tomaso et al., 2005), two colonies would fuse if they shared one allele at this locus. If this were the case, the parent and offspring should always fuse, as they share at least one allele at every locus. Siblings would also be expected to fuse with each other if they were produced via parthenogenesis or if both parents were homozygous at the colony-specificity locus. If the histoincompatibility loci in $P$. damicornis are not highly polymorphic, most siblings might fuse with each other, and many more sibling assays would be necessary to detect possible genetic differences 

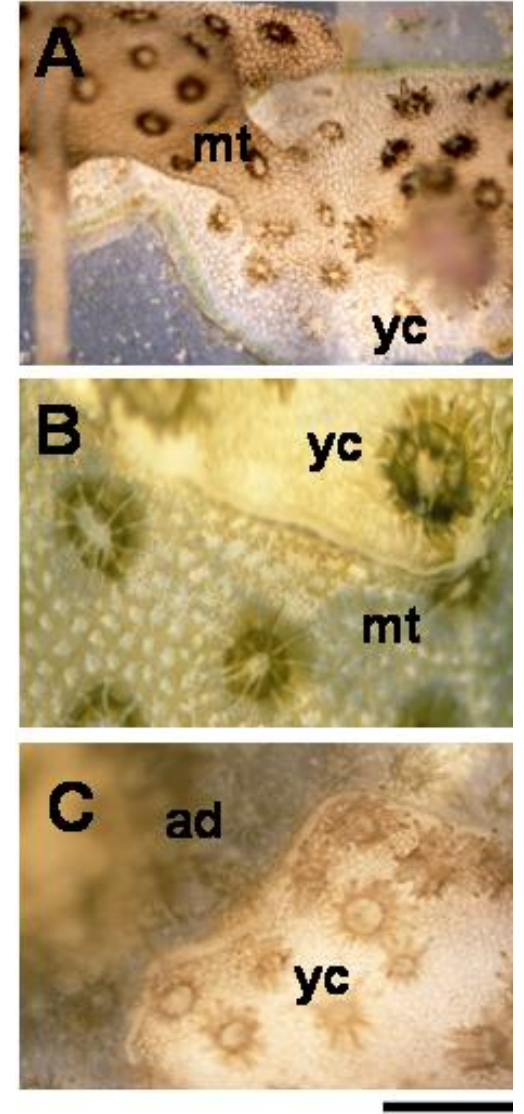

Figure 4. Contact reactions of Pocillopora damicornis parentoffspring pairs. A Fusion of a parent-offspring pair (MT5) 39 days after initial contact. B Incompatible fusion of a parent-offspring pair (PB07; 56 days). This is the only parent-offspring pair that showed incompatible fusion. C Non-fusion of a young colony (ZR3) and unrelated adult (PB07; 65 days). ad, adult; $\mathrm{mt}$, mother; yc, young colony. Scale bar $=20 \mathrm{~mm}$ for $\mathrm{A}$, $10 \mathrm{~mm}$ for $\mathrm{B}, \mathrm{C}$.

among siblings. Third, young colonies may lack functional histocompatibility systems, as suggested by Hidaka (1985) and Frank et al. (1997). The third possibility will be discussed further below.

In an early study on the histoincompatibility responses of $P$. damicornis, Hidaka (1985) observed that adult colonies displayed a histoincompatible response to allogeneic colonies, while primary polyps derived from different colonies fused with each other. He suggested that young polyps at an early stage of development lack a functional histoincompatibility system. A similar maturing of the histoincompatibility system has also been described in the coral Stylophora pistillata (Frank et al. 1997). Colonies younger than 2 months fused to form stable chimeras, but contacts between colonies 2-4 months old resulted in transient fusion, which terminated at the age of 4 months. After the age of 4 months, no fusion of allografts occurred. Nozawa and Loya (2005) also found that young colonies of Seriatopora caliendrum and S. hystrix fused only when grafted pairs were younger than 4 months old and that most fusion reactions transformed into nonfusion or incompatible fusion within that period. While Amar et al. (2008) proposed that sensitive early phases of ontogeny promote co-settlement of kin larvae of Stylophora pistillata to gain larger aggregates rapidly.

However, many young colony pairs of $P$. damicornis, S. caliendrum, S. hystrix and S. pistillata can show non-fusion and incompatible fusion responses at the age of 1 month or earlier (Hidaka et al., 1997; Nozawa and Loya, 2005; Amar et al., 2008; Figure 3 of this study). The genetic relationship between paired colonies, rather than the age of young colonies at contact, has been suggested to determine the contact response (Hidaka et al., 1997). Nozawa and Loya (2005) supported the idea that the genetic combination of grafts determines the contact reaction and its stability, although the degree of maturity of the allorecognition system may determine the onset and duration of both fusion and incompatible fusion. In our experiments, we observed the contact reactions
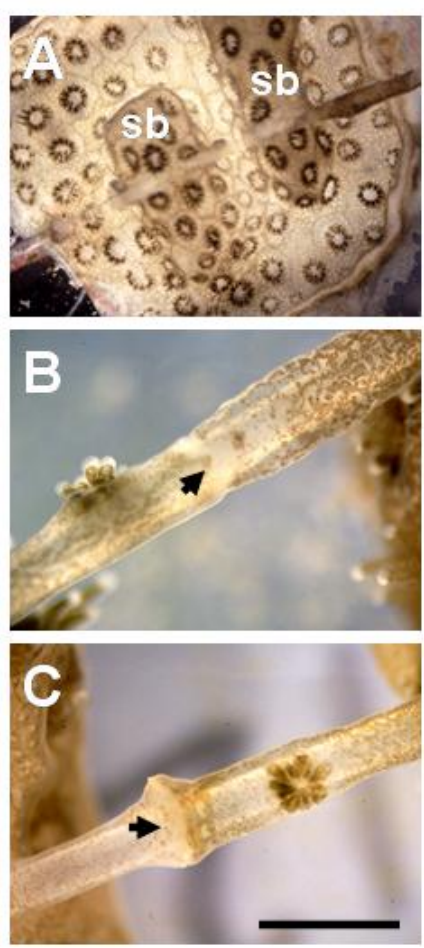

Figure 5. Contact reactions between allogeneic pairs of small branches isolated from adult Pocillopora damicornis colonies. A Fused pair from colonies PB13 and PB14 82 days after initial contact. $B$ Incompatible fusion of a pair from colonies ZR3 and PBO9 (39 days). Tissue contact occurred on a nylon thread, and a white zone without zooxanthellae can be observed (arrowhead). C Non-fused pair from colonies PB06-ZR4 (39 days). sb, small branch tips isolated from an adult colony. Scale bar $=30 \mathrm{~mm}$ for $\mathrm{A}, 10 \mathrm{~mm}$ for B, C. 
of young colonies for 6 months and found fusion between sibling colonies to be stable. Moreover, though the experiments were terminated after 6 months, sibling pairs remained fused after a year of observation. When fused one- and second- year old sibling were paired each other again, they showed fusion response as well (Permata and Hidaka, 2005). Thus the fusion of young sibling colonies is unlikely to be due to the immaturity of the histoincompatibility system. Still, we cannot rule out the possibility that pairs of sibling colonies develop a histoincompatible reaction more slowly, as suggested by Nozawa and Loya (2005). Indeed, Raymundo and Maypa (2004) found that fusion between P. damicornis siblings from Bantayan Reef, Philippines, was stable until 8 months after settlement, but at 8 to 9 months, some fused pairs displayed rejection responses.

Non-sibling pairs of young colonies derived from the same source colony combinations generally showed identical contact responses. However, some pairs from the same combination showed different responses. For example, one PB07-PB10 pair fused, while another five pairs did not. In three combinations involving colony ZR3, pairs appeared to fuse temporarily for different durations (Table 1). Two PB08-ZR3 pairs showed stable fusion, while the other four pairs transformed from fusion to incompatible fusion during the 6-month observation period. Two PB09-ZR3 pairs displayed a fusion response that later transformed to non-fusion, while the other two pairs displayed incompatible fusion from the start. In PB10-ZR3 combinations, three pairs fused, while one pair that was assayed 3 months after planulation showed incompatible fusion. Variations in the stability or duration of the fusion response among pairs from the same combinations involving colony ZR3 may indicate genetic differences among sibling colonies, although environmental factors could also modify the onset of delayed rejection.

When the outcomes of the three types of contact experiments (young-young, adult-young, and adult-adult pairs) were compared within the same combinations of source colonies, young colony pairs and pairs of their adult source colonies sometimes showed different contact responses (Table 4). In PB08-ZR3 and PB09-ZR3 combinations, adult pairs fused, while young colony pairs exhibited incompatible or temporary fusion. The differences cannot be due to the immature histocompatibility systems of young colonies, since adult source colony pairs were compatible. In PB07-PB08 combinations, two parent-offspring pairs showed fusion, while both pairs of young colonies and pairs of adult colonies displayed non-fusion. This result confirmed the dual modes of planulae production in $P$. damicornis
(Whitaker, 2006; Combosch and Vollmer, 2013). Although most planulae were asexually derived, however, some portions of planulae were sexually generated (Yeoh and Dai, 2009). More over recent report suggested that $P$. damicornis indeed consisted of 5 different species (Schmidt-Roach et al., 2014).

The high frequency of fusion between $P$. damicornis colonies collected from Bise and Motobu suggests that the degree of polymorphism at the histoincompatibility loci is markedly low in those areas. This is consistent with the report that asexual reproduction through fragmentation plays a major role in maintaining the $P$. damicornis population of the Ryukyu Archipelago (Adjeroud and Tsuchiya, 1999). The possibility that chimerism does occur in $P$ damicornis cannot be ruled out. Evidence reported from study of the species from Thailand and Philippines reefs. Chimeric larvae were observed through microsatellite marker. The chimeric larvae was thought derived asexually from mosaicked maternal colonies that was also a chimeric colonies (Rinkevich et al., 2016).

The increase of colonies size as the result of fusion, may enhance the benefits of chimerism in $P$. damicornis (Raymundo and Maypa, 2004). Similar conclusion was drawn that aggregated kin of $S$. pistillata which include fusing and rejecting partner gained benefit from its total size, though multipartner colonies tended to suffer higher cost of being chimera (Amar et al., 2008). Moreover, it is also likely that the genotypic diversity of $P$. damicornis has declined because of a mass coral bleaching event in 1998, during which branching corals, including $P$. damicornis, suffered a high mortality (Loya et al., 2001). Our results revealed no significant correlation between the frequency of the histoincompatibility reaction and the distance from each other of the two sites from which paired colonies were collected (Table 3). While colonies from Bise and Motobu ( $6 \mathrm{~km}$ from Bise) invariably failed to fuse with each other, some pairs of colonies collected from Bise and the Kerama Islands $(80 \mathrm{~km}$ apart) did fuse. However, this is not surprising, as the Kerama populations are considered as the larval source for Okinawa Island (Nishikawa et al., 2003).

In our study, when two colonies of $P$. damicornis come into contact, the contact reaction and the time needed for stable reactions are most probably under genetic control rather than influenced by maturity of histocompatibility system. Repeated contacts between the same pairs at different age, mostly yielded the same results. The finding that young colonies were able to recognize their partner less than 1 month old supports the idea. Further studies are necessary to observe more 
contact reaction between parent colonies and their offspring as well as between siblings to unveiled the genetic mechanism of histocompatibility in corals.

\section{Acknowledgments}

This study was partly supported by a Grant-inAid for Scientific Research $C$ from the Ministry of Science, Culture, Sports, and Education (Monbukagakusho) and a 21st century COE program of the University of the Ryukyus. D.P.W. also thanks the Japan Science Society for Sasakawa Scientific Research Grant financial support. The authors thank staff of Sesoko Station, Tropical Biosphere Research Center, where part of this study was done. We thank Dr. I. Yakovleva Dr. R. Bhagooli, and Dr. A. Nishikawa for assistance with field collection.

\section{References}

Adjeroud, M. \& Tsuchiya, M. 1999. Genetic variation and clonal structure in the scleractinian coral Pocillopora damicornis in the Ryukyu Archipelago, southern Japan. Mar. Biol. 134: 753-760. doi : $10.1007 /$ s002270050592

Amar, K. O., Chadwick, N. E. \& Rinkevich, B. 2008. Coral kin aggregations exhibit mixed allogeneic reactions and enhanced fitness during early ontogeny. BMC Evol. Biol. 8(1):126. doi: 10.1186/1471-2148-8-126

Amar, K. O., \& Rinkevich, B. 2010. Mounting of erratic histoincompatible responses in hermatypic corals: a multi-year interval comparison. J. Exp. Biol. 213(4):535-540. doi: 10.1242/jeb.039529

Ayre, D.J. \& Miller, K.J. 2004. Where do clonal coral larvae go? Adult genotypic diversity conflicts with reproductive effort in the brooding coral Pocillopora damicornis. Mar. Ecol. Prog. Ser., 277: 95-10

Barki, Y., Gateño, D., Graur, D., \& Rinkevich, B. 2002. Soft-coral natural chimerism: a window in ontogeny allows the creation of entities comprised of incongruous parts. Mar. Ecol. Prog. Ser. 231:91-99.

Cadavid, L.F., Powell, A.E., Nicotra, M.L., Moreno, M., \& Buss, L.W. 2004. An invertebrate histocompatibility complex. Genetics 167(1): 357-365. doi: 10.1534/genetics.167.1.357

Chadwick-Furman, N., \& Rinkevich, B. 1994. A complex allorecognition system in a reefbuilding coral: delayed responses, reversals and nontransitive hierarchies. Coral Reefs, 13(1):57-63.

Chornesky, E.A. 1991. The ties that bind: interclonal cooperation may help a fragile coral dominate shallow high-energy reefs. Mar. Biol. 109(1):41-51.

Combosch, D.J., \& Vollmer, S. V. 2013. Mixed asexual and sexual reproduction in the Indo-Pacific reef coral Pocillopora damicornis. Ecol. Evol. 3(10):3379-3387.

De Tomaso, A.W., Nyholm, S.V., Palmeri, K. J., Ishizuka, K.J., Ludington, W.B., Mitchel, K., \& Weissman, I.L. 2005. Isolation and characterization of a protochordate histocompatibility locus. Nature, 438(7067): 454-459. doi: 10.1038/nature04150

Frank, U., Oren, U., Loya, Y., \& Rinkevich, B. 1997. Alloimmune maturation in the coral Stylophora pistillata is achieved through three distinctive stages, 4 months post-metamorphosis. Proc. Royal Soc. London Biol. Sci. 264(1378):99-104. doi: $10.1098 /$ rspb.1997.0015

Grosberg, R.K. 1988. The Evolution of allorecognition specificity in clonal invertebrates. Q. Rev. Biol. 63:377-412.

Harrison, P., \& Wallace, C.C. 1990. Reproduction, dispersal, and recruitment of scleractinian corals. In Ecosystems of the world Vol. 25: Coral Reefs" Ed by Z Dubinsky, Elsevier Press, Amsterdam, pp 133-207

Hennige, S.J., Morrison, C.L., Form, A.U., Büscher, J., Kamenos, N.A., \& Roberts, J.M. 2014. Selfrecognition in corals facilitates deep-sea habitat engineering. Sci. Rep. 4:6782. doi : 10.1038/srep06782

Hidaka, M. 1985. Tissue compatibility between colonies and between newly settled larvae of Pocillopora damicornis. Coral Reefs 4(2):111116

Hidaka, M., Yurugi, K., Sunagawa, S., \& Kinzie lii, R.A. 1997. Contact reactions between young colonies of the coral Pocillopora damicornis. Coral Reefs. 16(1): 13-20.

Hildemann, W.H., Raison, R.L., Cheung, G., Hull, C.J., Akaka, L., \& Okamoto, J. 1977. Immunological specificity and memory in a scleractinian coral. Nature, 270(5634):219-301

Hildemann, W.H., Jokiel, P.L., Bigger, C.H., \& Johnston, I.S. 1980. Allogeneic polymorphism and alloimmune memory in the coral, 
Montipora verrucosa. Transplantation. 30:297301

Hunter, C.L. 1985. Assessment of clonal diversity and population structure of Porites compressa (Cnidaria, Scleractini). Proc 5th Coral reef Congr 6: 69-74

Loya, Y., Sakai, K., Yamazato, K., Nakano, Y., Sambali, H. \& van Woesik, R. 2001. Coral bleaching: the winners and losers. Ecol. Lett. 4(2):122-131

Miller, K.J., \& Ayre, D.J. 2004. The role of sexual and asexual reproduction in structuring high latitude populations of the reef coral Pocillopora damicornis. Heredity, 92(6):557-568 doi:10.1038/sj/hdy.6800459

Nishikawa, A., Katoh, M., \& Sakai, K. 2003. Larval settlement rates and gene flow of broadcastspawning (Acropora tenuis) and planulabrooding (Stylophora pistillata) corals. . Mar. Ecol. Prog. Ser.256: 87-97

Nozawa, Y. \& Loya, Y. 2005. Genetic relationship and maturity state of allorecognition system affect contact reactions in juvenile Seriatopora corals. Mar. Ecol. Prog. Ser. 286: 115-123

Nozawa, Y., \& Hirose, M. 2011. When does the window close?: The onset of allogeneic fusion 2-3 years post-settlement in the scleractinian coral, Echinophyllia aspera. Zool. Stud. 50:396

Oka, H., \& Watanabe, H. 1957. Colony-specificity in compound ascidians as tested by fusion experiments. Proc. Japan Acad. 33(10):657659.

Oka, H., \& Watanabe, H. 1960.) Problems of colony specificity in compound ascidians. Bull. Mar. Biol. Stn. Asamushi, 10: 153-155

Permata, W.D., Kinzie lii, R.A., \& Hidaka, M. 2000. Histological studies on the origin of planulae of the coral Pocillopora damicornis. Mar. Ecol. Prog. Ser. 200:191-200.

Permata, W.D., \& Hidaka, M. 2005. Ontogenetic changes in the capacity of the coral Pocillopora damicornis to originate branches. Zool. Sci. 22(11):1197-1203. doi;10.2108/zsj.22.1197

Puill-Stephan, E., Willis, B.L., Abrego, D., Raina, J.B., \& van Oppen, M.J.H. 2012. Allorecognition maturation in the broadcast-spawning coral Acropora millepora. Coral Reefs. 31(4):10191028.
Raymundo, L.J., \& Maypa, A.P. (2004). Getting bigger faster: mediation of size-specific mortality via fusion in juvenile coral transplants. Ecol. Appl. 14(1):281-295. doi: 10.1890/02-5373

Rinkevich, B., Shaish, L., Douek, J., \& Ben-Shlomo, R. 2016. Venturing in coral larval chimerism: a compact functional domain with fostered genotypic diversity. Sci. Rep. 6:19493. doi: 10.1038/srep19493

Schmidt-Roach S, Miller KJ, Woolsey E, Gerlach G, Baird, A.H. 2012 Broadcast spawning by Pocillopora species on the Great Barrier Reef. PLoS ONE 7(12): e50847. doi : 10.1371/journal.pone.0050847

Schmidt-Roach, S., Miller, K.J., Lundgren, P., \& Andreakis, N. 2014. With eyes wide open: a revision of species within and closely related to the Pocillopora damicornis species complex (Scleractinia; Pocilloporidae) using morphology and genetics. Zool. J. Linnean Soc. 170(1):133 doi: 10.1111/zoj.12092

Stoddart, J.A. 1983. Asexual production of planulae in the coral Pocillopora damicornis. Mar. Biol. 76(3):279-284.

Schweinsberg, M.R.A.G., Pech, R.G., Tollrian, R., \& Lampert, K.P. 2014. Transfer of intracolonial genetic variability through gametes in Acropora hyacinthus corals. Coral Reefs,.33(1):77-87. doi : $10.1007 / \mathrm{s} 00338-013-1102-5$

Veron, J.E.N \& Pichon, M. 1976. Scleractinian of eastern Australia. I. Families Thomasteriidae, Astrocoeniidae, Pocilloporidae. AIMS Monograph. Ser. 1: 88 pp

Ward, S. 1992. Evidence for broadcast spawning as well as brooding in the scleractinian coral Pocillopora damicornis. Mar. Biol. 112(4):641646

Whitaker, K. 2006. Genetic evidence for mixed modes of reproduction in the coral Pocillopora damicornis and its effect on population structure. Mar. Ecol. Prog. Ser. 306:115-124

Yeoh, S.R., \& Dai, C.F. 2010. The production of sexual and asexual larvae within single broods of the scleractinian coral, Pocillopora damicornis. Mar. Biol. 157(2): 351-359. doi : 10.1007/s00227-009-1322y 